\title{
A GENERAL MITCHELL STYLE ITERATION
}

\author{
JOHN KRUEGER
}

\begin{abstract}
We work out the details of a schema for a mixed support forcing iteration, which generalizes the Mitchell model [7] with no Aronszajn trees on $\omega_{2}$.
\end{abstract}

The main purpose of this paper is to present the details of a schema for a mixed support forcing iteration. This schema will provide the technical framework for a variety of consistency results which we establish in [6] and [4].

In [5] we constructed a model in which the properties of being "internally club" and "internally approachable" are distinct, for structures of size the successor of a regular cardinal. Our construction was reminiscent in some ways of Mitchell's classic model with no Aronszajn trees on $\omega_{2}([7])$. We used a forcing iteration whose factors are two-step iterations of Cohen forcing followed by a kind of collapse forcing, where the support on the Cohen part and on the collapsing part are of different sizes. However the kind of collapse forcing we used is not even strategically closed.

In Section 2 we describe an iterable property which is satisfied by both the forcing used to construct Mitchell's model and the forcing we used in [5]. It is this property which we will assume to hold for the factor forcings in our mixed support iteration schema. We work out the details of this iteration in Section 3.

In subsequent papers we use the iterated forcing schema presented below as a tool for establishing consistency results. In [6] we construct a model with a disjoint stationary sequence on the successor of an arbitrary regular cardinal, and also distinguish several variations of the property of being internally approachable for sets of a variety of sizes. In [4] we use the forcing iteration schema to study the approachability ideal at the second successor of a singular cardinal.

\section{Preliminaries}

We review some background material, notation, and conventions used in the paper. We assume the reader has had some previous exposure to proper forcing and iterated forcing. We refer the reader to [2] for basic facts concerning iterated forcing. In the rest of the section, $\mu$ and $\kappa$ always refer to regular cardinals.

A forcing poset is a pair $\langle\mathbb{P}, \leq\rangle$ such that $\leq$ is a binary relation on $\mathbb{P}$ which is reflexive and transitive. Due to our treatment of iterated forcing, we do not require anti-symmetry as part of the definition of a forcing poset. In the case that $p \leq q$ and $q \leq p$, we say that $p$ and $q$ are equivalent.

A forcing poset $\mathbb{P}$ is $\mu$-closed if whenever $\left\langle p_{i}: i<\xi\right\rangle$ is a descending sequence of conditions in $\mathbb{P}$ for some $\xi<\mu$, then there is $q$ in $\mathbb{P}$ such that $q \leq p_{i}$ for all $i<\xi$. The poset $\mathbb{P}$ satisfies the $\kappa$-covering property if it forces that whenever $x$ is a set of ordinals in the extension with size less than $\kappa$, then there is a set $a$ in the ground model with size less than $\kappa$ in the ground model such that $x \subseteq a$.

Date: April 2008. 
For a forcing poset $\mathbb{P}$ and an ordinal $\alpha$, we define a two-player game $G(\mathbb{P}, \alpha)$ as follows. Player I and Player II take turns to define a descending sequence $\left\langle p_{i}: 1 \leq\right.$ $i<\zeta\rangle$ of conditions in $\mathbb{P}$, with Player I playing $p_{j}$ for odd ordinals $j$ and Player II playing $p_{i}$ for even ordinals $i$. The game continues as long as possible, until either $p_{i}$ is defined for all $i<\alpha$, in which case Player II wins, or otherwise until Player II has no possible move, in which case Player I wins. Clearly Player I wins iff there is a limit ordinal $\delta<\alpha$ such that $\left\langle p_{i}: 1 \leq i<\delta\right\rangle$ has no lower bound. We say that $\mathbb{P}$ is $\alpha$-strategically closed if Player II has a winning strategy in the game $G(\mathbb{P}, \alpha)$.

The poset $\mathbb{P}$ is $<\kappa$-distributive if any family of fewer than $\kappa$ many dense open subsets of $\mathbb{P}$ has an intersection which is also dense open. This property is equivalent to $\mathbb{P}$ not adding any new sequences of ordinals with order type less than $\kappa$. If $\mathbb{P}$ is $\kappa$-closed then it is $\kappa$-strategically closed, and if $\mathbb{P}$ is $\kappa$-strategically closed then it is $<\kappa$-distributive. We say that $\mathbb{P}$ is $(<\kappa, \infty, \mu)$-distributive if for all $\xi<\kappa, \mathbb{P}$ forces that whenever $g: \xi \rightarrow O n$ is a function in the extension, then there is a function $h: \xi \rightarrow V$ in the ground model such that for all $i<\xi,|h(i)|^{V} \leq \mu$ and $g(i) \in h(i)$.

We use $\operatorname{ADD}(\mu)$ to denote the forcing poset which adds a Cohen subset to $\mu$. Conditions in $\operatorname{ADD}(\mu)$ are partial functions $f: \mu \rightarrow 2$ with domain of size less than $\mu$, and $q \leq p$ iff $q$ extends $p$ as a function. If $\mu^{<\mu}=\mu$ then $\operatorname{ADD}(\mu)$ has size $\mu$.

Consider a forcing iteration $\left\langle\mathbb{P}_{i}, \dot{\mathbb{Q}}_{j}: i \leq \alpha, j<\alpha\right\rangle$. We refer to the $\dot{\mathbb{Q}}_{j}$ 's as the factors of the iteration. In our notation, a condition in $\mathbb{P}_{\alpha}$ is a partial function $p: \alpha \rightarrow V$ whose domain is a subset of $\alpha$, and the support of $p$ is exactly the domain of $p$. We do not use the convention that $p(i)$ always denotes the maximal element of $\dot{\mathbb{Q}}_{i}$ when $i$ is not in the support of $p$. We also assume that whenever $p$ is a condition in $\mathbb{P}_{\alpha}$, then for all $i$ in the domain of $p, \mathbb{P}_{i}$ forces that $p(i)$ is in $\dot{\mathbb{Q}}_{i}$.

A useful fact about names in forcing is the so-called maximal principle, which asserts that whenever a condition $p$ in $\mathbb{P}$ forces $\exists x \varphi$, then there is a $\mathbb{P}$-name $\dot{a}$ such that $p$ forces $\varphi(\dot{a})$. We apply this fact in the paper in the following situation. Suppose $\left\langle\mathbb{P}_{i}, \dot{\mathbb{Q}}_{j}: i \leq \alpha, j<\alpha\right\rangle$ is a forcing iteration, $p$ and $q$ are conditions in $\mathbb{P}_{\alpha}$, and $q \leq p$. Then there is a condition $q^{\prime}$ in $\mathbb{P}_{\alpha}$ which is equivalent to $q$ such that for all $i$ in $\operatorname{dom}(p), \mathbb{P}_{i}$ forces that $q^{\prime}(i) \leq p(i)$. Let the domain of $q^{\prime}$ be equal to $\operatorname{dom}(q)$. If $i$ is in $\operatorname{dom}(q) \backslash \operatorname{dom}(p)$, let $q^{\prime}(i)=q(i)$. Suppose $i$ is in $\operatorname{dom}(p)$. Since $q\left\lceil i\right.$ forces $q(i) \leq p(i), \mathbb{P}_{i}$ forces the statement: $\exists x\left((x \leq p(i)) \&\left(q\left\lceil i \in \dot{G}_{i} \rightarrow\right.\right.\right.$ $x=q(i))$. By the maximal principle, let $q^{\prime}(i)$ be a $\mathbb{P}_{i}$-name $\dot{a}$ such that $\mathbb{P}_{i}$ forces $\left((\dot{a} \leq p(i)) \&\left(q \uparrow i \in \dot{G}_{i} \rightarrow \dot{a}=q(i)\right)\right.$. Then $q^{\prime}$ is a condition equivalent to $q$ and satisfies the desired property.

We use Even and Odd to denote the classes of even and odd ordinals respectively. If $X$ is a set, we say that a cardinal $\theta$ is much larger than $X$ if $\theta$ is larger than $|\mathcal{P}(\mathcal{P}(\mathcal{P}(\operatorname{tr}(X))))|$, where $\operatorname{tr}(X)$ is the transitive closure of $X$.

If $\mu^{<\mu}=\mu$, then the following statement, known as the $\Delta$-System Lemma, holds for $\mu$ : if $\left\{a_{i}: i<\mu^{+}\right\}$is a family of sets each of which has size less than $\mu$, then there is a set $a$ and an unbounded set $X \subseteq \mu^{+}$such that for $i<j$ in $X, a_{i} \cap a_{j}=a$.

Let $P_{\kappa}(X)$ denote the collection of subsets of $X$ which have size less than $\kappa$. Usually when this notation is used, $\kappa$ is a subset of $X$. A set $C \subseteq P_{\kappa}(X)$ is club in $P_{\kappa}(X)$ if it is closed under unions of $\subseteq$-increasing chains of length less than $\kappa$, and is cofinal in the sense that any member of $P_{\kappa}(X)$ is a subset of some member of $C$. A set $S \subseteq P_{\kappa}(X)$ is stationary in $P_{\kappa}(X)$ if it has non-empty intersection with each club set. 
We will be interested in elementary substructures of models which are an expansion of $\langle H(\lambda), \in\rangle$ in a countable language, where $\lambda \geq \omega_{2}$ is a regular cardinal. Suppose $N \prec H(\lambda)$ and $N \cap \kappa \in \kappa$. Then any set $x$ in $N$ with size less than $\kappa$ is a subset of $N$. For $|x| \in N$ by elementarity, and by the fact that $N \cap \kappa \in \kappa,|x| \subseteq N$. Choose $f:|x| \rightarrow x$ a bijection in $N$. Then $f[|x|]=x \subseteq N$.

Let $N$ be a set and $\xi$ a limit ordinal. We say that $N$ is internally approachable of length $\xi$, and is in $I A(\xi)$, if there is a $\subseteq$-increasing and continuous sequence of sets $\left\langle N_{i}: i<\xi\right\rangle$ with union equal to $N$ such that for all $j<\xi,\left\langle N_{i}: i<j\right\rangle$ is in $N$. We say that $N$ is internally approachable if it is internally approachable of length some limit ordinal.

An internally approachable chain is a $\subseteq$-increasing and continuous sequence of sets $\left\langle N_{i}: i<\xi\right\rangle$, where $\xi$ is a limit ordinal, such that for all $j<\xi,\left\langle N_{i}: i \leq j\right\rangle \in$ $N_{j+1}$. Note that the union of an internally approachable chain of length $\xi$ is in $I A(\xi)$. Conversely, suppose $\kappa<\lambda$ are regular uncountable cardinals, and $\mathcal{A}$ is an expansion of $H(\lambda)$ in a countable language. Assume that $N \prec \mathcal{A}, N \cap \kappa \in \kappa, \kappa \in N$, and $N$ is internally approachable. Then there exists an internally approachable chain $\left\langle N_{i}: i<\xi\right\rangle$ with union $N$, for some limit ordinal $\xi$, such that for all $i<\xi$, $N_{i} \prec \mathcal{A}$ and $N_{i} \cap \kappa \in \kappa$. If in addition $N$ is in $I A(\mu)$ for a regular cardinal $\mu$, then there is such an internally approachable chain which has length $\mu$. See Section 2 of [3] for more information about internally approachable sets.

Suppose $N \prec\langle H(\lambda), \in, \mathbb{P}\rangle$. A condition $q$ in $\mathbb{P}$ is $N$-generic for $\mathbb{P}$ if for any dense open subset $D$ of $\mathbb{P}$ in $N, q$ forces that $\dot{G} \cap N \cap D$ is non-empty. This implies that $q$ forces $N[\dot{G}] \cap V=N$, and in particular, $N[\dot{G}] \cap O n=N \cap O n$ (in fact, these properties are equivalent for $q$ ). For suppose $G$ is a generic filter containing $q$, and let $b$ be in $N[G] \cap V$. Fix a $\mathbb{P}$-name $\dot{c}$ in $N$ such that $\dot{c}^{G}=b$. Let $D$ be the dense open set of conditions which either force $\dot{c}$ is not in $V$, or decide the value of $\dot{c}$. By elementarity, $D$ is in $N$. Fix $s$ in $G \cap N \cap D$. Since $b$ is in $V, s$ decides the value of $\dot{c}$. Then $b$ is in $N$, since it is definable from $s$ and $\dot{c}$.

We use the following generalization of proper forcing. Let $\mathbb{P}$ be a forcing poset, and let $\mu<\kappa$ be regular cardinals. We say that $\mathbb{P}$ is $<\kappa$-proper for $I A(\mu)$ if for all sufficiently large regular cardinals $\theta>\kappa$ which are much larger than $\mathbb{P}$, there is a club $C \subseteq P_{\kappa}(H(\theta))$ such that for any $N$ in $C \cap I A(\mu)$, for all $p$ in $N \cap \mathbb{P}$ there is $q \leq p$ which is $N$-generic.

\section{FACtors of the Iteration}

In [8] Mitchell gives a modern description of the construction from [7] of a model with no Aronszajn trees on $\omega_{2}$, as an iteration of adding Cohen reals and $\omega_{1}$-closed collapses up to a weakly compact cardinal, with finite support on the Cohen forcings and countable support on the collapses. In [5] we used a similar kind of mixed support iteration to construct a model in which the notions of internally club and internally approachable are distinct. We iterated alternately adding Cohen subsets to a regular cardinal $\mu$ and then collapsing $\mu^{++}$to have size $\mu^{+}$, with supports of size less than $\mu$ on the Cohen forcings and supports with size $\mu$ on the collapses. However, the kind of collapses we used are not even strategically closed.

Thus we sought an iterable property which is satisfied by the forcing posets used in both constructions. Let $\mu^{<\mu}=\mu$ be a cardinal, and let $\kappa>\mu$ be regular. We consider two-step iterations $\operatorname{ADD}(\mu) * \dot{\mathbb{Q}}$, equipped with two partial orderings. The first partial ordering $\leq$ is the usual ordering on a two-step iteration. The second 
ordering $\leq^{*}$ is defined by letting $q * \dot{t} \leq * p * \dot{s}$ if $q * \dot{t} \leq p * \dot{s}$ and $q=p$. It turns out that the desired property is:

The poset $\left\langle\operatorname{ADD}(\mu) * \dot{\mathbb{Q}}, \leq^{*}\right\rangle$ is $\kappa$-strategically closed.

As we see next, if $\operatorname{ADD}(\mu)$ forces that $\dot{\mathbb{Q}}$ is $\kappa$-strategically closed, then the above property holds. However, we will show at the end of the section that this property does not imply that $\operatorname{ADD}(\mu)$ forces $\dot{\mathbb{Q}}$ is $\kappa$-strategically closed.

Proposition 2.1. If $\operatorname{ADD}(\mu)$ forces $\dot{\mathbb{Q}}$ is $\kappa$-strategically closed, then $\langle\operatorname{ADD}(\mu) * \dot{\mathbb{Q}}, \leq *$ \rangle is $\kappa$-strategically closed. In particular, if $\operatorname{ADD}(\mu)$ forces that $\dot{\mathbb{Q}}$ is $\kappa$-closed then $\left\langle\operatorname{ADD}(\mu) * \dot{\mathbb{Q}}, \leq^{*}\right\rangle$ is $\kappa$-strategically closed.

Proof. Let $\dot{\sigma}$ be an $\operatorname{ADD}(\mu)$-name for a strategy for Player II in the game $G(\dot{\mathbb{Q}}, \kappa)$. Define a strategy $\tau$ for Player II in the game $G\left(\left\langle\operatorname{ADD}(\mu) * \dot{\mathbb{Q}}, \leq^{*}\right\rangle, \kappa\right)$ as follows. Let Player I begin the game with a condition $p_{1} * \dot{q}_{1}$. Suppose a play $\left\langle p_{j} * \dot{q}_{j}: 1 \leq j<i\right\rangle$ is defined where $i<\kappa$ is even. By the definition of $\leq^{*}, p_{j}=p_{1}$ for all $1 \leq j<i$. Assume as an induction hypothesis that $p_{1}$ forces $\left\langle\dot{q}_{j}: 1 \leq j<i\right\rangle$ is a play of the game $G(\dot{\mathbb{Q}}, \kappa)$ according to $\dot{\sigma}$. Let $\tau$ instruct Player II to play a condition $p * \dot{q}_{i}$ such that $p$ forces $\dot{q}_{i}$ is Player II's response to $\left\langle\dot{q}_{j}: 1 \leq j<i\right\rangle$ according to $\dot{\sigma}$. Thus Player II is able to pass through every limit stage less than $\kappa$ and wins the game.

The next proposition describes the forcing poset we used in [5].

Proposition 2.2. Let $\lambda \geq \kappa$ be a regular cardinal. Let $\operatorname{ADD}(\mu) * \dot{\mathbb{Q}}$ be the twostep iterated forcing defined as follows. If $G$ is generic for $\operatorname{ADD}(\mu)$, then in $V[G]$, $\dot{\mathbb{Q}}^{G}$ is the forcing poset whose conditions are increasing and continuous sequences $\left\langle a_{i}: i \leq \gamma\right\rangle$ of sets such that $\gamma<\kappa$ and $a_{i}$ is in $P_{\kappa}\left(H(\lambda)^{V}\right) \cap V$ for $i \leq \gamma$, ordered by extension of sequences. Then $\left\langle\operatorname{ADD}(\mu) * \dot{\mathbb{Q}}, \leq^{*}\right\rangle$ is $\kappa$-strategically closed.

Proof. Fix a regular cardinal $\theta$ which is much larger than $\operatorname{ADD}(\mu) * \dot{\mathbb{Q}}$, and let $\mathcal{A}$ denote the structure $\langle H(\theta), \in, \operatorname{ADD}(\mu) * \dot{\mathbb{Q}}, \lambda\rangle$. We describe a strategy $\sigma$ for Player II in the game $G\left(\left\langle\operatorname{ADD}(\mu) * \dot{\mathbb{Q}}, \leq^{*}\right\rangle, \kappa\right)$ by describing his or her moves on a play $\left\langle p * \dot{q}_{i}: 1 \leq i<\kappa\right\rangle$. Simultaneously we define an increasing and continuous sequence $\left\langle N_{i}: i<\kappa\right\rangle$ of elementary substructures of $\mathcal{A}$ with size less than $\kappa$. To begin with fix $N_{0} \prec \mathcal{A}$ with size less than $\kappa$ such that $N_{0} \cap \kappa \in \kappa$ and $\mu \subseteq N_{0}$. Let $p * \dot{q}_{1}$ be Player I's first move.

Suppose that $i<\kappa$ and we have defined $\left\langle p * \dot{q}_{j}: 1 \leq j \leq i\right\rangle$ and $\left\langle N_{j}: j<i\right\rangle$. If $i$ is a limit ordinal let $N_{i}=\bigcup\left\{N_{j}: j<i\right\}$. Otherwise let $N_{i}$ be an elementary substructure of $\mathcal{A}$ with size less than $\kappa$ such that $N_{i-1} \subseteq N_{i}, N_{i} \cap \kappa \in \kappa$, and $p * \dot{q}_{i}$ is in $N_{i}$. If $i$ is even, let $p * \dot{q}_{i+1}$ be Player I's next move.

Suppose $i$ is odd. Let $\sigma$ instruct Player II to play a condition $p * \dot{q}_{i+1}$, where $p$ forces that $\dot{q}_{i+1}$ is a sequence with domain equal to $\operatorname{dom}\left(\dot{q}_{i}\right)+1$, such that $\dot{q}_{i+1}\left\lceil\operatorname{dom}\left(\dot{q}_{i}\right)=\dot{q}_{i}\right.$ and $\dot{q}_{i+1}\left(\operatorname{dom}\left(\dot{q}_{i}\right)\right)=N_{i} \cap H(\lambda)^{V}$. Clearly $N_{i} \cap H(\lambda)^{V}$ is in $P_{\kappa}\left(H(\lambda)^{V}\right) \cap V$. So to show that this definition makes sense, we need to check $p$ forces that the maximal element of $\dot{q}_{i}$ is a subset of $N_{i} \cap H(\lambda)^{V}$. Let $G$ be generic for $\operatorname{ADD}(\mu)$. Then $N_{i}[G] \cap V=N_{i}$, since $\operatorname{ADD}(\mu) \subseteq N_{i}$. Now $q_{i}=\dot{q}_{i}^{G}$ is a member of $N_{i}[G]$. Let $a$ be the last element on the sequence $q_{i}$, which is in $N_{i}[G]$ by elementarity. Since $|a|<\kappa, a \subseteq N_{i}[G] \cap H(\lambda)^{V}=N_{i} \cap H(\lambda)^{V}$. 
Now suppose $\delta<\kappa$ is a limit ordinal and we have defined $\left\langle p * \dot{q}_{j}: 1 \leq j<\delta\right\rangle$ and $\left\langle N_{j}: j<\delta\right\rangle$. Let $N_{\delta}=\bigcup\left\{N_{i}: i<\delta\right\}$. Let $\dot{q}_{\delta}^{*}$ be a name for $\bigcup\left\{\dot{q}_{i}: i<\delta\right\}$, and let $\dot{\gamma}$ be a name for the length of this sequence. Then by construction, for all odd $i<\delta, p$ forces that $N_{i} \cap H(\lambda)^{V}$ is the maximal element of the sequence $\dot{q}_{i+1}$. Therefore $p$ forces that the union of the sequence $\dot{q}_{\delta}^{*}$ is equal to $\bigcup\left\{N_{i} \cap H(\lambda)^{V}: i \in\right.$ $\delta \cap \operatorname{Odd}\}=N_{\delta} \cap H(\lambda)^{V}$, which is in $P_{\kappa}\left(H(\lambda)^{V}\right) \cap V$. So let $\sigma$ instruct Player II to play the condition $p * \dot{q}_{\delta}$ such that $p$ forces $\dot{q}_{\delta} \uparrow \dot{\gamma}=\dot{q}_{\delta}^{*}$ and $\dot{q}_{\delta}(\dot{\gamma})=N_{\delta} \cap H(\lambda)^{V}$. Thus Player II is able to pass through all limit stages less than $\kappa$, and wins the game.

We give an example to show that $\left\langle\operatorname{ADD}(\mu) * \dot{\mathbb{Q}}, \leq^{*}\right\rangle$ being $\kappa$-strategically closed does not imply that $\operatorname{ADD}(\mu)$ forces that $\dot{\mathbb{Q}}$ is $\kappa$-strategically closed.

Let $\mu=\omega$ and $\kappa=\omega_{1}$, and let $\operatorname{ADD}(\omega) * \dot{\mathbb{Q}}$ be the forcing poset described in Proposition 2.2 letting $\lambda=\omega_{2}$. We show that $\operatorname{ADD}(\omega)$ forces that $\dot{\mathbb{Q}}$ is not $\omega_{1}$-strategically closed.

Let $G$ be a generic filter for $\operatorname{ADD}(\omega)$ over $V$. We use the fact due to Abraham and Shelah [1] that in $V[G]$, the set $P_{\omega_{1}}\left(H\left(\omega_{2}\right)^{V}\right) \backslash V$ is stationary in $P_{\omega_{1}}\left(H\left(\omega_{2}\right)^{V}\right)$. Suppose for a contradiction that there is a strategy $\sigma$ for the game $G\left(\mathbb{Q}, \omega_{1}\right)$ in $V[G]$. Working in $V[G]$, let $\theta$ be a regular cardinal much larger than $\omega_{2}$ and let $\mathcal{A}$ be the structure $\langle H(\theta), \in, \mathbb{Q}, \sigma\rangle$. Fix a countable $N \prec \mathcal{A}$ such that $N \cap H\left(\omega_{2}\right)^{V}$ is not in $V$. Fix an enumeration $\left\langle x_{n}: n<\omega\right\rangle$ of $N \cap H\left(\omega_{2}\right)^{V}$.

We consider a partial run of the game $\left\langle p_{j}: 1 \leq j \leq \omega\right\rangle$ defined as follows. We will arrange that $p_{j}$ is in $N$ for all $j<\omega$. Let Player I begin the game by playing a condition $p_{1}$ in $N$ such that $x_{0}$ is in the maximal element of $p_{1}$. Suppose $\left\langle p_{j}: 1 \leq j \leq i\right\rangle$ is defined where $1 \leq i<\omega$. If $i=2 n$ is even, let Player I choose some $p_{i+1} \leq p_{i}$ in $\mathbb{Q} \cap N$ such that the maximal element of $p_{i+1}$ contains $x_{n}$ as an element. If $i$ is odd, let Player II play $p_{i+1}$ according to $\sigma$. Since $\sigma$ is in $N, p_{i+1}$ is in $N$. This defines $\left\langle p_{i}: i<\omega\right\rangle$. Let $p_{\omega}$ be Player II's response to this run of the game according to $\sigma$.

Let $\bigcup\left\{p_{n}: n<\omega\right\}=\left\langle a_{i}: i<\gamma\right\rangle$ and let $a=\bigcup\left\{a_{i}: i<\gamma\right\}$. For each $n<\omega$, $p_{n}$ is in $N$. Since $p_{n}$ is countable, $p_{n} \subseteq N$. So for $i<\gamma, a_{i}$ is in $N$, and hence $a_{i} \subseteq N$ since $a_{i}$ is countable. Therefore $a \subseteq N \cap H\left(\omega_{2}\right)^{V}$. On the other hand, if $z$ is in $N \cap H\left(\omega_{2}\right)^{V}$, then $z=x_{n}$ for some $n$, and so for some $i<\gamma, z$ is in $a_{i}$. Hence $a=N \cap H\left(\omega_{2}\right)^{V}$. But then $p_{\omega}(\gamma)$ is equal to $N \cap H\left(\omega_{2}\right)^{V}$, since $p_{\omega}$ is a continuous sequence. By the definition of $\mathbb{Q}, N \cap H\left(\omega_{2}\right)^{V}$ is in $V$, which is a contradiction.

Here is another way of viewing this argument, pointed out by the referee. The poset $\operatorname{ADD}(\omega)$ adds stationarily many new countable subsets of $H\left(\omega_{2}\right)^{V}$. The poset $\dot{\mathbb{Q}}$ then adds a club set which is disjoint from this stationary set, thus destroying its stationarity. If $\dot{\mathbb{Q}}$ is $\omega_{1}$-strategically closed, then it is proper. But proper forcing posets cannot destroy the stationarity of stationary collections of countable sets.

\section{Forcing Iteration with Mixed Support}

We describe a general schema for a mixed support forcing iteration. Fix a regular cardinal $\mu$ such that $\mu^{<\mu}=\mu$ and let $\kappa$ be a regular cardinal greater than $\mu$ such that for all $\zeta<\kappa, \zeta^{<\mu}<\kappa$.

Let us consider a forcing iteration

$$
\left\langle\mathbb{P}_{i}, \dot{\mathbb{Q}}_{j}: i \leq \alpha, j<\alpha\right\rangle,
$$


with two partial orderings $\leq$ and $\leq^{*}$. The iteration will alternate between forcing with $\operatorname{ADD}(\mu)$ at even stages, and other posets at odd stages. The iteration will be $\mu$-closed, and therefore the poset $\operatorname{ADD}(\mu)$ is the same in both the ground model and in any intermediate extension. Specifically, suppose the iteration satisfies the following description.

(A) If $i<\alpha$ is even, $\mathbb{P}_{i}$ forces $\dot{\mathbb{Q}}_{i}=\operatorname{ADD}(\mu)$.

(B) If $i<\alpha$ then $\mathbb{P}_{i}$ forces $\dot{\mathbb{Q}}_{i}$ is $\mu$-closed.

(C) If $j<\alpha$ is odd, then $\mathbb{P}_{j-1}$ forces that $\left\langle\dot{\mathbb{Q}}_{j-1} * \dot{\mathbb{Q}}_{j}, \leq^{*}\right\rangle=\left\langle\operatorname{ADD}(\mu) * \dot{\mathbb{Q}}_{j}, \leq^{*}\right\rangle$ is $\kappa$-strategically closed.

(D) If $i \leq \alpha$ is a limit ordinal, $\mathbb{P}_{i}$ consists of all partial functions $p: i \rightarrow V$ such that $p\left\lceil j \in \mathbb{P}_{j}\right.$ for $j<i,|\operatorname{dom}(p) \cap \operatorname{Even}|<\mu$, and $|\operatorname{dom}(p) \cap \operatorname{Odd}|<\kappa$.

(E) For $i \leq \alpha$ and $p$ and $q$ in $\mathbb{P}_{i}, q \leq p$ in $\mathbb{P}_{i}$ iff for all $\gamma$ in the domain of $p, \gamma$ is in the domain of $q$ and $q\lceil\gamma \Vdash q(\gamma) \leq p(\gamma)$.

(F) For $i \leq \alpha$ and $p$ and $q$ in $\mathbb{P}_{i}, q \leq^{*} p$ in $\mathbb{P}_{i}$ iff $q \leq p$, $\operatorname{dom}(p) \cap$ Even $=$ $\operatorname{dom}(q) \cap$ Even, and for all $\gamma$ in $\operatorname{dom}(p) \cap$ Even, $q\lceil\gamma$ forces $q(\gamma)=p(\gamma)$.

We will prove that such an iteration preserves all cardinals and cofinalities less than or equal to $\kappa$. Hence by recursion, conditions (A), (B), and (C) make sense. Also, since such an iteration is $\mu$-closed, the cardinal arithmetic assumptions we made in the ground model, that $\mu^{<\mu}=\mu$ and $\zeta^{<\mu}<\kappa$ for all $\zeta<\kappa$, will remain true in the extension.

For $\beta \leq \alpha$, let $\mathbb{P}_{\beta}^{\prime}$ be the subset of $\mathbb{P}_{\beta}$ consisting of conditions $p$ such that for all even $i$ in the domain of $p$, there is $x$ in $\operatorname{ADD}(\mu)$ such that $p(i)$ is the canonical $\mathbb{P}_{i}$-name for $x$. Note that if $\beta<\gamma \leq \alpha$, then $\mathbb{P}_{\beta}^{\prime} \subseteq \mathbb{P}_{\gamma}^{\prime}$.

We make a list of the properties which we prove $\mathbb{P}_{\alpha}$ satisfies.

(I) $\left\langle\mathbb{P}_{\alpha}, \leq\right\rangle$ is $\mu$-closed.

(II) $\left\langle\mathbb{P}_{\alpha}, \leq^{*}\right\rangle$ is $\kappa$-strategically closed.

(III) $\mathbb{P}_{\alpha}^{\prime}$ is a $\mu$-closed dense subset of $\mathbb{P}_{\alpha}$.

(IV) $\mathbb{P}_{\alpha}$ is $<\kappa$-proper for $I A(\mu)$.

(V) If $p$ is in $\mathbb{P}_{\alpha}^{\prime}$ and $p \Vdash \dot{\beta}$ is an ordinal, then there is $q \leq^{*} p$ and a set $x$ in $V$ with size less than or equal to $\mu$ such that $q \Vdash \dot{\beta} \in \check{x}$.

(VI) $\mathbb{P}_{\alpha}$ is $(<\kappa, \infty, \mu)$-distributive.

(VII) $\mathbb{P}_{\alpha}$ preserves all cardinals and cofinalities less than or equal to $\kappa$.

(VIII) For every regular cardinal $\lambda$ less than or equal to $\kappa, \mathbb{P}_{\alpha}$ preserves the stationarity of all stationary subsets of $\lambda$.

(IX) $\mathbb{P}_{\alpha}$ forces that whenever $X \subseteq V$ and for all $A$ in $\left([V]^{<\kappa}\right)^{V}, X \cap A \in V$, then $X$ is in $V$. 
The most difficult properties to verify are (IV), (V), and (IX), so we prove these last. Properties (I), (II), and (III) have fairly standard proofs.

Lemma 3.1. The posets $\left\langle\mathbb{P}_{\alpha}, \leq\right\rangle$ and $\left\langle\mathbb{P}_{\alpha}^{\prime}, \leq\right\rangle$ are $\mu$-closed.

Proof. Suppose $\left\langle p_{i}: i<\xi\right\rangle$ is a descending sequence in $\mathbb{P}_{\alpha}$, where $\xi<\mu$ is a limit ordinal. We define a lower bound $q$ in $\mathbb{P}_{\alpha}$ with domain equal to $X=\bigcup\left\{\operatorname{dom}\left(p_{i}\right)\right.$ : $i<\xi\}$. Suppose $q\left\lceil\gamma\right.$ is defined and is below $p_{i} \uparrow \gamma$ for all $i<\xi$. If $\gamma$ is in $X$, then $q\left\lceil\gamma\right.$ forces that $\left\langle p_{i}(\gamma): i<\xi, \gamma \in \operatorname{dom}\left(p_{i}\right)\right\rangle$ is a descending sequence in $\dot{\mathbb{Q}}_{\gamma}$. By property (B), choose a $\mathbb{P}_{\gamma}$-name $q(\gamma)$ for a lower bound of this sequence. If each $p_{i}$ is in $\mathbb{P}_{\alpha}^{\prime}$, then also make sure that for all even $\gamma$ in $X, q(\gamma)$ is the canonical $\mathbb{P}_{\gamma}$-name for the union of $\left\{p_{i}(\gamma): \gamma \in \operatorname{dom}\left(p_{i}\right)\right\}$.

Lemma 3.2. The poset $\left\langle\mathbb{P}_{\alpha}, \leq^{*}\right\rangle$ is $\kappa$-strategically closed.

Proof. By property (C), for each odd $\gamma+1<\alpha$, let $\dot{\sigma}_{\gamma}$ be a $\mathbb{P}_{\gamma}$-name for a strategy for Player II in the game $G\left(\left\langle\operatorname{ADD}(\mu) * \dot{\mathbb{Q}}_{\gamma+1}, \leq^{*}\right\rangle, \kappa\right)$. Define a strategy $\sigma$ for Player II in the game $G\left(\left\langle\mathbb{P}_{\alpha}, \leq^{*}\right\rangle, \kappa\right)$ as follows. Suppose a partial play $\left\langle p_{i}: 1 \leq i<\zeta\right\rangle$ of the game is given where $\zeta$ is even. Let $X=\bigcup\left\{\operatorname{dom}\left(p_{i}\right): 1 \leq i<\zeta\right\}$. For each odd $\gamma+1$ in $X$, let $i_{\gamma}$ be the least ordinal $i<\zeta$ such that $\gamma+1 \in \operatorname{dom}\left(p_{i}\right)$. Assume inductively that for each odd $\gamma+1$ in $X, i_{\gamma}$ is odd, and for all $i_{\gamma} \leq j<\zeta, p_{j}\lceil\gamma$ forces that $\left\langle p_{k}(\gamma) * p_{k}(\gamma+1): i_{\gamma} \leq k \leq j\right\rangle$ enumerates a partial play in the game $G\left(\left\langle\operatorname{ADD}(\mu) * \dot{\mathbb{Q}}_{\gamma+1}, \leq^{*}\right\rangle, \kappa\right)$ according to $\dot{\sigma}_{\gamma}$, where we interpret $p_{k}(\gamma)$ to be $1_{\mathrm{ADD}(\mu)}$ if $\gamma$ is not in $\operatorname{dom}\left(p_{k}\right)$. Now let $\sigma$ instruct Player II to play the condition $p_{\zeta}$ with domain equal to $X$ such that $p_{\zeta} \uparrow$ Even $=p_{1} \uparrow$ Even and for all odd $\gamma+1$ in $X$, $p_{\zeta}\left\lceil\gamma\right.$ forces $p_{\zeta}(\gamma) * p_{\zeta}(\gamma+1)$ is Player II's response to $\left\langle p_{k}(\gamma) * p_{k}(\gamma+1): i_{\gamma} \leq k<\zeta\right\rangle$ according to $\dot{\sigma}_{\gamma}$.

Lemma 3.3. For $\beta \leq \alpha, \mathbb{P}_{\beta}^{\prime}$ is a dense subset of $\mathbb{P}_{\beta}$.

Proof. We prove the statement by induction on $\beta$. Note that for all $\beta \leq \alpha, \mathbb{P}_{\beta}$ satisfies (A) - (F), so by Lemma $3.1,\left\langle\mathbb{P}_{\beta}, \leq\right\rangle$ and $\left\langle\mathbb{P}_{\beta}^{\prime}, \leq\right\rangle$ are $\mu$-closed. Suppose $\beta \leq \alpha$ and for all $\gamma<\beta, \mathbb{P}_{\gamma}^{\prime}$ is dense in $\mathbb{P}_{\gamma}$. Let $p$ in $\mathbb{P}_{\beta}$ be given.

First assume $\beta$ is a successor ordinal $\gamma+1$. If $\gamma$ is not in the domain of $p$, then $p$ is in $\mathbb{P}_{\gamma}$, so by the induction hypothesis choose $q \leq p$ in $\mathbb{P}_{\gamma}^{\prime}$. Then $q \leq p$ in $\mathbb{P}_{\beta}$ and $q$ is in $\mathbb{P}_{\beta}^{\prime}$. Otherwise first find $p^{\prime} \leq p\left\lceil\gamma\right.$ in $\mathbb{P}_{\gamma}^{\prime}$ such that for some $x$ in $\operatorname{ADD}(\mu)$, $p^{\prime}$ forces $p(\gamma)=\check{x}$. This is possible by the induction hypothesis and the fact that since $\mathbb{P}_{\gamma}$ is $\mu$-closed, $\mathbb{P}_{\gamma}$ forces $\operatorname{ADD}(\mu)$ is the same poset in both the ground model and the extension. Then $p^{\prime \frown} \check{x} \leq p$ and $p^{\prime \frown} \check{x}$ is in $\mathbb{P}_{\beta}^{\prime}$.

Assume now that $\beta$ is a limit ordinal. If $\operatorname{cf}(\beta) \geq \mu$, then by property (D) there is $\xi<\beta$ such that $\operatorname{dom}(p) \cap$ Even $\subseteq \xi$. By the induction hypothesis, find $q \leq p\lceil\xi$ in $\mathbb{P}_{\xi}^{\prime}$. Then $q^{\wedge}\left(p\lceil[\xi, \beta))\right.$ is a refinement of $p$ in $\mathbb{P}_{\beta}^{\prime}$.

Now suppose $\operatorname{cf}(\beta)<\mu$. Fix an increasing and continuous sequence $\left\langle\xi_{i}: i<\right.$ $\operatorname{cf}(\beta)\rangle$ cofinal in $\beta$ with $\xi_{0}=0$, and let $\xi_{\operatorname{cf}(\beta)}=\beta$. Define by induction a descending sequence $\left\langle p_{i}: i \leq \operatorname{cf}(\beta)\right\rangle$, with $p_{0}=p$, so that $p_{i}\left\lceil\xi_{i}\right.$ is in $\mathbb{P}_{\xi_{i}}^{\prime}$. Given $p_{i}$, apply the induction hypothesis and choose $q \leq p_{i}\left\lceil\xi_{i+1}\right.$ in $\mathbb{P}_{\xi_{i+1}}^{\prime}$, and let $p_{i+1}=q$ ^ $(p \uparrow$ $\left.\left[\xi_{i+1}, \beta\right)\right)$. Suppose $\delta \leq \operatorname{cf}(\beta)$ is a limit ordinal and $p_{i}$ is defined for all $i<\delta$. For each $i<\delta, p_{i}\left\lceil\xi_{i}\right.$ is in $\mathbb{P}_{\xi_{i}}^{\prime}$, and $\mathbb{P}_{\xi_{i}}^{\prime}$ is a subset of $\mathbb{P}_{\xi_{\delta}}^{\prime}$. Since $\mathbb{P}_{\xi_{\delta}}^{\prime}$ is $\mu$-closed, let $q$ be a lower bound of $\left\langle p_{i}\left\lceil\xi_{i}: i<\delta\right\rangle\right.$ in $\mathbb{P}_{\xi_{\delta}}^{\prime}$. We claim that $q$ is below $p_{i}\left\lceil\xi_{\delta}\right.$ for $i<\delta$. So let $i<\delta$. Since $\xi_{\delta}$ is a limit ordinal, it suffices to show that $q \leq p_{i}\lceil\zeta$ for all $\zeta<\xi_{\delta}$. Given $\zeta<\xi_{\delta}$, choose $i<j<\delta$ large enough so that $\zeta \leq \xi_{j}$. Then 
$q \leq p_{j}\left\lceil\xi_{j} \leq p_{j}\left\lceil\zeta \leq p_{i}\left\lceil\zeta\right.\right.\right.$. Now let $p_{\delta}=q \widehat{q}\left(p\left\lceil\left[\xi_{\delta}, \beta\right)\right)\right.$. This completes the construction. The condition $p_{\operatorname{cf}(\beta)}$ is a refinement of $p$ in $\mathbb{P}_{\beta}^{\prime}$.

This completes the proof of properties (I), (II), and (III).

Since the proofs of properties (IV) and (V) are complicated, let us first show that property (V) implies properties (VI), (VII), and (VIII). So assume that whenever $p$ is in $\mathbb{P}_{\alpha}^{\prime}$ and $p$ forces $\dot{\beta}$ is an ordinal, there is $q \leq^{*} p$ and a set $x$ with size less than or equal to $\mu$ such that $q$ forces $\dot{\beta}$ is in $\check{x}$. Note that this implies the following property:

$\left(\mathrm{V}^{\prime}\right)$ If $p$ is in $\mathbb{P}_{\alpha}^{\prime}, q \leq^{*} p$, and $q \Vdash \dot{\beta}$ is an ordinal, then there is $r \leq^{*} q$ and a set $x$ with size less than or equal to $\mu$ such that $r \Vdash \dot{\beta} \in \check{x}$.

The point is, if $p$ is in $\mathbb{P}_{\alpha}^{\prime}$ and $q \leq^{*} p$, then there is $q^{\prime}$ in $\mathbb{P}_{\alpha}^{\prime}$ which is equivalent to $q$. Namely, let $q^{\prime} \uparrow$ Odd $=q \uparrow$ Odd and $q^{\prime} \uparrow$ Even $=p\lceil$ Even. Now apply (V) to $q^{\prime}$ and $\dot{\beta}$.

Fix for the remainder of the section a winning strategy $\sigma$ for Player II in the game $G\left(\left\langle\mathbb{P}_{\alpha}, \leq^{*}\right\rangle, \kappa\right)$.

Lemma 3.4. $\left\langle\mathbb{P}_{\alpha}, \leq\right\rangle$ is $(<\kappa, \infty, \mu)$-distributive.

Proof. Let $\xi<\kappa$ be a limit ordinal, and suppose $p$ is a condition in $\mathbb{P}_{\alpha}^{\prime}$ which forces that $\dot{f}: \xi \rightarrow O n$ is a function. We define a partial play $\left\langle p_{i}: 1 \leq i \leq \xi\right\rangle$ in the game $G\left(\left\langle\mathbb{P}_{\alpha}, \leq^{*}\right\rangle, \kappa\right)$. Let Player I play $p_{1}=p$. Suppose $\left\langle p_{i}: 1 \leq i<\gamma\right\rangle$ is defined. If $\gamma$ is even, let $p_{\gamma}$ be Player II's response according to $\sigma$. Suppose $\gamma=2 \cdot \zeta+1$ is odd. Applying $\left(\mathrm{V}^{\prime}\right)$, let Player I choose $p_{\gamma} \leq^{*} p_{\gamma-1}$ such that for a set $x_{\zeta}$ with size less than or equal to $\mu, p_{\gamma}$ forces $\dot{f}(\zeta)$ is in $\check{x}_{\zeta}$. This completes the construction. Let $q=p_{\xi}$, and define $h: \xi \rightarrow V$ by $h(i)=x_{i}$. Then $q$ forces $\dot{f}(i) \in h(i)$ for all $i<\xi$.

We note that (VIII) implies (VII). For suppose in some generic extension $V[G]$, there is an ordinal $\xi \leq \kappa$ which changed its cofinality. Assume $\xi$ is the least such ordinal. In $V[G], \operatorname{cf}(\xi)=\operatorname{cf}\left(\operatorname{cf}^{V}(\xi)\right)$. So by the minimality of $\xi, \operatorname{cf}^{V}(\xi)=\xi$, and $\xi$ is regular in $V$. Suppose $\operatorname{cf}(\xi)=\beta$ in $V[G]$. Then $\beta$ is regular in both $V$ and $V[G]$. Since $\beta<\xi$, the set $S=\xi \cap \operatorname{cof}(\beta)$ is stationary in $\xi$ in $V$. But in $V[G]$ there is a club in $\xi$ with order type $\beta$, and the limits points of this club have cofinality less than $\beta$. Thus $S$ becomes non-stationary in $V[G]$, contradicting (VIII). Now if any cardinals are collapsed, the first collapsed cardinal would have to be a successor cardinal, which would change its cofinality. So indeed (VIII) implies (VII).

Proposition 3.5. For all regular $\lambda \leq \kappa$ and any stationary set $S \subseteq \lambda, \mathbb{P}_{\alpha}$ forces $S$ is stationary.

Proof. If $\lambda<\mu$ then $\mathbb{P}_{\alpha}$ does not even add any subsets to $\lambda$. If $\lambda=\mu, \mathbb{P}_{\alpha}$ preserves stationary subsets of $\lambda$ since it is $\mu$-closed. So assume $\mu<\lambda \leq \kappa$.

Suppose $p$ is in $\mathbb{P}_{\alpha}^{\prime}$ and $p$ forces $\dot{C}$ is club in $\lambda$. We find $q \leq p$ which forces $S \cap \dot{C}$ is non-empty. We define a sequence $\left\langle p_{i}, \beta_{i}, x_{i}: 1 \leq i<\lambda\right\rangle$, where the sequence of $p_{i}$ 's constitutes a partial play of the game $G\left(\left\langle\mathbb{P}_{\alpha}, \leq^{*}\right\rangle, \kappa\right)$ according to $\sigma$. Let $p_{1}=p, \beta_{1}=0$, and $x_{1}=\emptyset$.

Suppose $\left\langle p_{i}, \beta_{i}, x_{i}: 1 \leq i<\gamma\right\rangle$ is defined for some $\gamma<\lambda$, where $\left\langle\beta_{i}: 1 \leq i<\gamma\right\rangle$ is an increasing and continuous sequence of ordinals less than $\lambda$. If $\gamma$ is even, let $p_{\gamma}$ 
be Player II's response according to $\sigma$ to the sequence of conditions defined thus far. Then let $\beta_{\gamma}=\sup \left(\left\{\beta_{i}+1: 1 \leq i<\gamma\right\}\right)$ and $x_{\gamma}=\emptyset$.

Suppose $\gamma$ is odd. Applying $\left(\mathrm{V}^{\prime}\right)$, choose $p_{\gamma} \leq^{*} p_{\gamma-1}$ and a set $x_{\gamma}$ with size less than or equal to $\mu$ such that $p_{\gamma}$ forces $\min \left(\dot{C} \backslash \beta_{\gamma-1}\right) \in \check{x}_{\gamma}$. Now choose an ordinal $\beta_{\gamma}$ in $\lambda$ larger than both $\beta_{\gamma-1}$ and $\sup \left(x_{\gamma}\right)$.

By construction the sequence $\left\langle\beta_{i}: 1 \leq i<\lambda\right\rangle$ is strictly increasing and continuous, and so clearly is cofinal in $\lambda$. Since $S$ is stationary, fix a limit ordinal $\delta<\lambda$ such that $\beta_{\delta}$ is in $S$. For every odd $\gamma<\delta, p_{\gamma}$ forces $\dot{C} \cap\left[\beta_{\gamma-1}, \beta_{\gamma}\right)$ is non-empty. Therefore $p_{\delta}$ forces $\beta_{\delta}$ is a limit point of $\dot{C}$, and hence is in $\dot{C} \cap S$.

We have proven properties (VI), (VII), and (VIII), assuming (V). It remains to show (IV), (V), and (IX).

The following basic lemma is helpful in the remaining proofs.

Lemma 3.6. Suppose $p$ and $q$ are conditions in $\mathbb{P}_{\alpha}$ such that for all $\gamma$ in $\operatorname{dom}(p) \cap$ $\operatorname{dom}(q)$, either $p\left\lceil\gamma\right.$ or $q\left\lceil\gamma\right.$ forces $p(\gamma)$ and $q(\gamma)$ are compatible in $\dot{\mathbb{Q}}_{\gamma}$. Then $p$ and $q$ are compatible.

Proof. Define $u$ below $p$ and $q$ by inductively defining $u\lceil\gamma$ for $\gamma<\alpha$. Assume $u\lceil\gamma$ is defined and is a condition below $p\lceil\gamma$ and $q \uparrow \gamma$. If $\gamma$ is not in $\operatorname{dom}(p) \cup \operatorname{dom}(q)$, then $\gamma$ is not in the domain of $u$. If $\gamma$ is in $\operatorname{dom}(p) \backslash \operatorname{dom}(q)$, let $u(\gamma)=p(\gamma)$, and if $\gamma$ is in $\operatorname{dom}(q) \backslash \operatorname{dom}(p)$, let $u(\gamma)=q(\gamma)$. Suppose $\gamma$ is in $\operatorname{dom}(p) \cap \operatorname{dom}(q)$. By assumption and the induction hypothesis, we can fix a $\mathbb{P}_{\gamma}$-name $u(\gamma)$ such that $u\lceil\gamma$ forces $u(\gamma) \leq p(\gamma), q(\gamma)$.

Proposition 3.7. Let $\theta$ be a regular cardinal much larger than $\mathbb{P}_{\alpha}$, and let $\mathcal{A}$ denote the structure $\left\langle H(\theta), \in,<_{\theta}, \mathbb{P}_{\alpha}, \sigma\right\rangle$, where $<_{\theta}$ is a well-ordering of $H(\theta)$. Suppose $N$ is an elementary substructure of $\mathcal{A}$ in $P_{\kappa}(H(\theta)), N \cap \kappa \in \kappa$, and $N$ is internally approachable. Let $p$ be in $N \cap \mathbb{P}_{\alpha}^{\prime}$. Then:

(1) There is $q \leq^{*} p$ such that for any dense subset $D$ of $\left\langle\mathbb{P}_{\alpha}, \leq^{*}\right\rangle$ in $N$, there is $u$ in $N \cap D$ such that $q \leq^{*} u$.

(2) If, in addition, $N$ is in $I A(\mu)$, then there is $q \leq^{*} p$ which is $N$-generic for the forcing $\left\langle\mathbb{P}_{\alpha}, \leq\right\rangle$.

In particular, $\left\langle\mathbb{P}_{\alpha}, \leq\right\rangle$ is $<\kappa$-proper for $I A(\mu)$.

Conclusion (1) in the proposition asserts the existence of a strongly $N$-generic condition for the poset $\mathbb{P}_{\alpha}$ with the weak ordering $\leq^{*}$. Conclusion (2) asserts the existence of an $N$-generic condition for the poset $\mathbb{P}_{\alpha}$ with the usual ordering $\leq$. The proof of (1) is an easy variation of the standard technique for constructing generic conditions for closed forcings over internally approachable models.

Proof. (1) Let $p$ be in $N \cap \mathbb{P}_{\alpha}^{\prime}$. Fix an internally approachable chain $\left\langle N_{i}: i<\xi\right\rangle$, where $\xi<\kappa$ is a limit ordinal, with union $N$, such that $p$ is in $N_{0}$, and for $i<\xi$, $N_{i}$ is an elementary substructure of $\mathcal{A}$ with $N_{i} \cap \kappa \in \kappa$. Write $N=N_{\xi}$. Define by induction a sequence $\left\langle p_{i}: 1 \leq i \leq \xi\right\rangle$ which is partial play in the game $G\left(\left\langle\mathbb{P}_{\alpha}, \leq^{*}\right.\right.$ \rangle$, \kappa)$, such that for all $\gamma \leq \xi,\left\langle p_{i}: 1 \leq i<\gamma\right\rangle$ is definable in $\mathcal{A}$ from $\left\langle N_{i}: i<\gamma\right\rangle$ and $p$. Let $p_{1}=p$.

Suppose $\left\langle p_{i}: 1 \leq i<\gamma\right\rangle$ is defined as required. If $\gamma$ is even, let $p_{\gamma}$ be Player II's response to this sequence according to $\sigma$. By the induction hypothesis, clearly $\left\langle p_{i}: 1 \leq i<\gamma+1\right\rangle$ is definable in $\mathcal{A}$ from $\left\langle N_{i}: i<\gamma+1\right\rangle$ and $p$ as required. Assume $\gamma$ is odd. Since $\left\langle\mathbb{P}_{\alpha}, \leq^{*}\right\rangle$ is $\kappa$-strategically closed, it is $<\kappa$-distributive. 
But $\left|N_{\gamma-1}\right|<\kappa$. So let $p_{\gamma}$ be the $<_{\theta}$-least $\leq{ }^{*}$-refinement of $p_{\gamma-1}$ which is in the intersection of all dense open subsets of $\left\langle\mathbb{P}_{\alpha}, \leq^{*}\right\rangle$ in $N_{\gamma-1}$. Clearly $\left\langle p_{i}: 1 \leq i<\gamma+1\right\rangle$ is definable in $\mathcal{A}$ from $\left\langle N_{i}: i<\gamma+1\right\rangle$ and $p$. Now if $\left\langle p_{i}: 1 \leq i<\delta\right\rangle$ is defined for a limit ordinal $\delta \leq \xi$, the definition given above witnesses that this sequence is definable in $\mathcal{A}$ from $\left\langle N_{i}: i<\delta\right\rangle$ and $p$.

This completes the construction of $\left\langle p_{i}: 1 \leq i \leq \xi\right\rangle$. Every proper initial segment of the sequence $\left\langle p_{i}: 1 \leq i<\xi\right\rangle$ is definable in $\mathcal{A}$ from $p$ and a proper initial segment of $\left\langle N_{i}: i<\xi\right\rangle$, and so is in $N$. In particular, $p_{i}$ is in $N$ for all $i<\xi$. Now let $q=p_{\xi}$. By construction, for every dense open subset $D$ of $\left\langle\mathbb{P}_{\alpha}, \leq^{*}\right\rangle$ in $N$, there is $i<\xi$ such that $p_{i}$ is in $N \cap D$, and $q \leq^{*} p_{i}$. This implies the conclusion of (1).

(2) Now suppose in addition that $N$ is in $I A(\mu)$. Then we can take $\xi=\mu$ in the proof of (1). It follows that $N^{<\mu} \subseteq N$. For if $y$ is a subset of $N$ with size less than $\mu$, there is $i<\mu$ such that $y \subseteq N_{i}$. But by assumption on $\kappa,\left|\left[N_{i}\right]<\mu\right|<\kappa$. Since $N \cap \kappa \in \kappa$, this implies $\left[N_{i}\right]^{<\mu} \subseteq N$. So $y$ is in $N$.

Recall that $p$ is a condition in $N \cap \mathbb{P}_{\alpha}^{\prime}$. By (1), we can fix $q \leq^{*} p$ such that for any dense subset $D$ of $\left\langle\mathbb{P}_{\alpha}, \leq^{*}\right\rangle$ in $N$, there is $u$ in $N \cap D$ such that $q \leq^{*} u$. Suppose for a contradiction that $q$ is not $N$-generic for $\left\langle\mathbb{P}_{\alpha}, \leq\right\rangle$. Then there is a dense open subset $E$ of $\left\langle\mathbb{P}_{\alpha}, \leq\right\rangle$ in $N$ such that $q$ does not force $N \cap E \cap \dot{G}$ is non-empty. Fix $r \leq q$ in $\mathbb{P}_{\alpha}^{\prime}$ which forces that $N \cap E \cap \dot{G}$ is empty. It follow that for any $y$ in $N \cap E$, $r$ and $y$ are incompatible. Let $g: \alpha \rightarrow \operatorname{ADD}(\mu)$ be the partial function such that $\operatorname{dom}(g)=\operatorname{dom}(r) \cap$ Even $\cap N$ and for all $i$ in $\operatorname{dom}(g), r(i)$ is the canonical $\mathbb{P}_{i}$-name for $g(i)$. By property (D), $g$ has size less than $\mu$. For all $i$ in the domain of $g, i$ is in $N$, and so $\mathbb{P}_{i}$ is in $N$. Since $\operatorname{ADD}(\mu) \subseteq N$, it follows that $g(i)$ is in $N$. Since $N^{<\mu} \subseteq N, g$ is in $N$.

Let $D$ be the set of $u$ in $\mathbb{P}_{\alpha}$ such that either $u$ is $\leq^{*}$-incompatible with $p$, or $u \leq^{*} p$ and there is $y \leq u$ in $E \cap \mathbb{P}_{\alpha}^{\prime}$ such that:

(i) $\operatorname{dom}(g) \subseteq \operatorname{dom}(y)$, and for all $i$ in $\operatorname{dom}(g), y(i)$ is the canonical $\mathbb{P}_{i}$-name for a condition in $\operatorname{ADD}(\mu)$ which extends $g(i)$,

(ii) $y \uparrow \operatorname{Odd}=u \uparrow$ Odd

Note that by elementarity $D$ is in $N$.

We claim that $D$ is dense in $\left\langle\mathbb{P}_{\alpha}, \leq^{*}\right\rangle$. So let $s$ in $\mathbb{P}_{\alpha}$ be given. If $s$ is $\leq^{*}$ incompatible with $p$, then $s$ is in $D$. Otherwise fix $t \leq^{*} p, s$.

Define $w$ by letting $w \uparrow \operatorname{Odd}=t \uparrow \operatorname{Odd}, \operatorname{dom}(w) \cap$ Even $=\operatorname{dom}(g)$, and for all $i$ in $\operatorname{dom}(g), w(i)$ is the canonical $\mathbb{P}_{i}$-name for $g(i)$. We claim $w$ is a condition below $t$. Since $p \in N$ and $|\operatorname{dom}(p)|<\kappa, \operatorname{dom}(p) \subseteq N$. But $r \leq p$ and $\operatorname{dom}(r) \cap \operatorname{Even} \cap N=$ $\operatorname{dom}(g)$. So $\operatorname{dom}(p) \cap$ Even $\subseteq \operatorname{dom}(g)$. Since $t \leq^{*} p, \operatorname{dom}(t) \cap$ Even $=\operatorname{dom}(p) \cap$ Even. Hence $\operatorname{dom}(t) \subseteq \operatorname{dom}(w)$. Suppose $w \uparrow \gamma$ is a condition below $t \uparrow \gamma$ where $\gamma$ is in the domain of $w$, and we show that $w \uparrow \gamma+1$ is a condition below $t \uparrow \gamma+1$. If $\gamma$ is odd then $w(\gamma)=t(\gamma)$. Suppose $\gamma$ is even. If $\gamma$ is not in the domain of $t$ then there is nothing to check. Otherwise $\gamma$ is in $\operatorname{dom}(p)$. Since $r \leq p, r \uparrow \gamma$ forces $r(\gamma) \leq p(\gamma)$. But this implies $g(\gamma)$ is below the condition in $\operatorname{ADD}(\mu)$ named by $p(\gamma)$. Since $t \uparrow \gamma$ forces $p(\gamma)=t(\gamma), w \uparrow \gamma$ forces $w(\gamma) \leq t(\gamma)$.

Now extend $w$ to $y$ which is in $E \cap \mathbb{P}_{\alpha}^{\prime}$. Without loss of generality we can assume that for all odd $\gamma$ in $\operatorname{dom}(t), \mathbb{P}_{\gamma}$ forces $y(\gamma) \leq t(\gamma)$; for if $y$ did not already have this property, there is a condition equivalent to $y$ which does. Now define $u$ so that $u \uparrow$ Even $=t \uparrow$ Even and $u \uparrow$ Odd $=y \uparrow$ Odd. Then clearly $y \leq u \leq{ }^{*} t$. So $u \leq \leq^{*} s$, and $y$ witnesses that $u$ is in $D$; namely, (ii) is by definition, and (i) follows from the fact that $y$ is in $\mathbb{P}_{\alpha}^{\prime}$ and $y \leq w$. 
Since $D$ is in $N$ and is dense in $\left\langle\mathbb{P}_{\alpha}, \leq^{*}\right\rangle$, by (1) there is $u$ in $N \cap D$ such that $q \leq^{*} u$. Since $q \leq^{*} u, p, u$ is $\leq^{*}$-compatible with $p$. So by the definition of $D$ and elementarity, there is $y \leq u$ in $E \cap \mathbb{P}_{\alpha}^{\prime} \cap N$ satisfying (i) and (ii) above. Since $y$ is in $N \cap E$, by assumption $r$ and $y$ are incompatible. We will get a contradiction by showing that $r$ and $y$ are in fact compatible.

If $r$ and $y$ are incompatible, then by Lemma 3.6 let $\gamma$ be the least ordinal in $\operatorname{dom}(r) \cap \operatorname{dom}(y)$ such that $r \uparrow \gamma$ does not force $r(\gamma)$ and $y(\gamma)$ are compatible. Note that $\operatorname{dom}(y) \subseteq N$ since $|\operatorname{dom}(y)|<\kappa$. So $\gamma$ is in $N$. If $\gamma$ is even then $\gamma$ is in $\operatorname{dom}(r) \cap$ Even $\cap N=\operatorname{dom}(g)$. Then by $(\mathrm{i}), \mathbb{P}_{\gamma}$ forces $y(\gamma)$ is below $r(\gamma)$, which is impossible. So $\gamma$ is odd. But then by (ii), $u(\gamma)=y(\gamma)$. Since $r \leq q \leq u, r \uparrow \gamma$ forces $r(\gamma) \leq u(\gamma)=y(\gamma)$, which is again impossible. So $r$ and $y$ are compatible.

Thus we have proven property (IV).

Corollary 3.8. The poset $\mathbb{P}_{\alpha}$ has the $\kappa$-covering property. In fact, if $p$ is in $\mathbb{P}_{\alpha}^{\prime}$ and $p$ forces $\dot{a}$ is a set of ordinals with size less than $\kappa$, then there is $q \leq^{*} p$ and a set $x$ with size less than $\kappa$ such that $q \Vdash \dot{a} \subseteq \check{x}$.

Proof. Suppose $p$ is in $\mathbb{P}_{\alpha}^{\prime}$ and $p$ forces $\dot{a}$ is a set of ordinals with size less than $\kappa$. Let $\theta$ be a regular cardinal much larger than $\mathbb{P}_{\alpha}$ and $\dot{a}$, and let $\mathcal{A}$ denote the structure $\left\langle H(\theta), \in,<_{\theta}, \mathbb{P}_{\alpha}, \sigma\right\rangle$, where $<_{\theta}$ is a well-ordering of $H(\theta)$. Fix an elementary substructure $N$ of $\mathcal{A}$ such that $|N|<\kappa, N \cap \kappa \in \kappa, p$ and $\dot{a}$ are in $N$, and $N$ is in $I A(\mu)$. Applying Proposition 3.7(2), let $q \leq^{*} p$ be $N$-generic. Let $x=N \cap O n$. If $G$ is a generic filter for $\mathbb{P}_{\alpha}$ which contains $q$, then $N[G] \cap O n=$ $N \cap O n=x$. In particular, $N[G] \cap \kappa=N \cap \kappa \in \kappa$. Since $a=\dot{a}^{G}$ has size less than $\kappa$ and $N[G] \prec H(\theta)^{V[G]}, a \subseteq N[G] \cap O n=x$. So $q$ forces $\dot{a} \subseteq \check{x}$.

Proposition 3.9. If $p$ is in $\mathbb{P}_{\alpha}^{\prime}$ and $p$ forces $\dot{\beta}$ is an ordinal, then there is $q \leq^{*} p$ and a set $x$ with size less than or equal to $\mu$ such that $q \Vdash \dot{\beta} \in \check{x}$.

Proof. If $\kappa=\mu^{+}$then the statement follows from Corollary 3.8, letting $\dot{a}=\{\dot{\beta}\}$. So assume $\kappa>\mu^{+}$.

Suppose for a contradiction that there is no $q \leq^{*} p$ and $x$ as in the conclusion of the proposition. We define a sequence $\left\langle p_{i}, q_{i}, \beta_{i}: 1 \leq i<\mu^{+}\right\rangle$, where the $p_{i}$ 's constitute a partial play in $G\left(\left\langle\mathbb{P}_{\alpha}, \leq^{*}\right\rangle, \kappa\right)$ according to $\sigma$, and the $q_{i}$ 's are in $\mathbb{P}_{\alpha}^{\prime}$. Let $p_{1}=p, \beta_{1}=0$, and $q_{i}=1_{\mathbb{P}_{\alpha}}$. Suppose $\gamma<\mu^{+}$is even and for all $1 \leq i<\gamma, p_{i}$, $\beta_{i}$, and $q_{i}$ are defined. Let $p_{\gamma}$ be Player II's response to the play $\left\langle p_{i}: 1 \leq i<\gamma\right\rangle$ according to $\sigma$, and let $\beta_{\gamma}=0$ and $q_{\gamma}=1_{\mathbb{P}_{\alpha}}$.

Suppose $2 \leq \gamma<\mu^{+}$is even and $p_{i}, \beta_{i}$, and $q_{i}$ are defined for all $1 \leq i \leq \gamma$. Since $p_{\gamma} \leq^{*} p$, by assumption $p_{\gamma}$ does not force that $\dot{\beta}$ is in the set $\left\{\beta_{i}: 1 \leq i \leq \gamma\right\}$. So we can fix $q_{\gamma+1} \leq p_{\gamma}$ in $\mathbb{P}_{\alpha}^{\prime}$ and an ordinal $\beta_{\gamma+1}$ not in the set $\left\{\beta_{i}: 1 \leq i \leq \gamma\right\}$ such that $q_{\gamma+1}$ forces $\dot{\beta}=\beta_{\gamma+1}$. Moreover, choose $q_{\gamma+1}$ to have the property that for all $i$ in $\operatorname{dom}\left(p_{\gamma}\right) \cap \operatorname{Odd}, \mathbb{P}_{i}$ forces $q_{\gamma+1}(i) \leq p_{\gamma}(i)$. Now define $p_{\gamma+1}$ by letting $p_{\gamma+1} \uparrow$ Even $=p_{\gamma} \uparrow$ Even and $p_{\gamma+1} \uparrow$ Odd $=q_{\gamma+1} \uparrow$ Odd. Clearly $p_{\gamma+1} \leq{ }^{*} p_{\gamma}$.

Since $\kappa>\mu^{+}$, let $q$ be Player II's response to the play $\left\langle p_{i}: 1 \leq i<\mu^{+}\right\rangle$according to $\sigma$. Note that $\left\{q_{i}: i \in \mu^{+} \cap \operatorname{Odd}\right\}$ is an antichain. For if $i<j<\mu^{+}$are odd, then $\beta_{i} \neq \beta_{j}, q_{i} \Vdash \dot{\beta}=\check{\beta}_{i}$, and $q_{j} \Vdash \dot{\beta}=\check{\beta}_{j}$. Using the fact that $\mu^{<\mu}=\mu$, apply the $\Delta$-System Lemma to the family $\left\{\operatorname{dom}\left(q_{i}\right) \cap\right.$ Even $\left.: i \in \mu^{+} \cap \operatorname{Odd}\right\}$ to find an unbounded set $X \subseteq \mu^{+} \cap$ Odd and a set $a$ such that for $i<j$ in $X$, $\operatorname{dom}\left(q_{i}\right) \cap \operatorname{dom}\left(q_{j}\right) \cap$ Even $=a$. Since $a$ has size less than $\mu, \operatorname{ADD}(\mu)$ has size $\mu$, and each $q_{i}$ is in $\mathbb{P}_{\alpha}^{\prime}$, there are distinct $1<i<j$ in $X$ such that $q_{i} \uparrow a=q_{j} \uparrow a$. 
We get a contradiction by showing that $q_{i}$ and $q_{j}$ are compatible. Otherwise, by Lemma 3.6 let $\gamma$ be least such that $\gamma$ is in $\operatorname{dom}\left(q_{i}\right) \cap \operatorname{dom}\left(q_{j}\right)$ and $q_{j}\lceil\gamma$ does not force $q_{i}(\gamma)$ and $q_{j}(\gamma)$ are compatible. Clearly $\gamma$ is not even, since otherwise it is in $a$ and therefore $q_{i}(\gamma)=q_{j}(\gamma)$. So $\gamma$ is odd. By construction, $q_{i}(\gamma)=p_{i}(\gamma)$. But $q_{j} \leq p_{j-1} \leq p_{i}$, so $q_{j}\left\lceil\gamma\right.$ forces $q_{j}(\gamma) \leq p_{i}(\gamma)=q_{i}(\gamma)$, which is not possible. So indeed $q_{i}$ and $q_{j}$ are compatible, giving us a contradiction.

This completes the proof of property (V). It remains only to prove property (IX). We use the following lemma.

Lemma 3.10. Let $p$ and $q$ be conditions in $\mathbb{P}_{\alpha}^{\prime}$ and $t$ a condition in $\mathbb{P}_{\alpha}$ such that $p, q \leq t$. Then there are $p^{\prime} \leq p$ and $q^{\prime} \leq q$ in $\mathbb{P}_{\alpha}^{\prime}$ such that $p^{\prime} \uparrow$ Odd $=q^{\prime} \uparrow$ Odd and for all odd $i$ in $\operatorname{dom}(t), \mathbb{P}_{i}$ forces $p^{\prime}(i)=q^{\prime}(i)$ is below $t(i)$.

Proof. Without loss of generality assume $p$ satisfies the property that whenever $i$ is an odd ordinal in $\operatorname{dom}(t), \mathbb{P}_{i}$ forces $p(i) \leq t(i)$, since otherwise we can replace $p$ with an equivalent condition which does satisfy this property. We also assume the same for $q$. Now define $p^{\prime}$ and $q^{\prime}$ as follows. First choose $p^{\prime}(0) \leq p(0)$ and $q^{\prime}(0) \leq q(0)$ in $\operatorname{ADD}(\mu)$ so that $p^{\prime}(0)$ and $q^{\prime}(0)$ are incompatible. Suppose $\beta>0$ is an even ordinal and $p^{\prime} \uparrow \beta$ and $q^{\prime} \uparrow \beta$ are defined. Let $\beta$ be in $\operatorname{dom}\left(p^{\prime}\right)$ iff $\beta$ is in $\operatorname{dom}(p)$, in which case let $p^{\prime}(\beta)=p(\beta)$, and similarly with $q^{\prime}$. Suppose $\gamma$ is odd and $p^{\prime}\left\lceil\gamma\right.$ and $q^{\prime} \uparrow \gamma$ are defined. If $\gamma$ is not in $\operatorname{dom}(p) \cup \operatorname{dom}(q)$ then $\gamma$ is not in the domain of either $p^{\prime}$ or $q^{\prime}$. If $\gamma$ is in $\operatorname{dom}(p) \backslash \operatorname{dom}(q)$ then let $p^{\prime}(\gamma)=q^{\prime}(\gamma)=p(\gamma)$, and if $\gamma$ is in $\operatorname{dom}(q) \backslash \operatorname{dom}(p)$ then let $p^{\prime}(\gamma)=q^{\prime}(\gamma)=q(\gamma)$. Suppose $\gamma$ is in $\operatorname{dom}(p) \cap \operatorname{dom}(q)$. Let $\dot{x}_{\gamma}$ be a $\mathbb{P}_{\gamma}$-name such that $\mathbb{P}_{\gamma}$ forces " $\dot{x}_{\gamma}=p(\gamma)$ if $p^{\prime}\lceil\gamma$ is in $\dot{G}_{\gamma}$, and $\dot{x}_{\gamma}=q(\gamma)$ otherwise". Then $\dot{x}_{\gamma}$ is well-defined because $p^{\prime}\left\lceil\gamma\right.$ and $q^{\prime}\lceil\gamma$ are incompatible, due to the choice of $p^{\prime}(0)$ and $q^{\prime}(0)$. Let $p^{\prime}(\gamma)=q^{\prime}(\gamma)=\dot{x}_{\gamma}$.

Proposition 3.11. The poset $\mathbb{P}_{\alpha}$ forces that whenever $X \subseteq V$ and for all $A$ in $\left([V]^{<\kappa}\right)^{V}, X \cap A \in V$, then $X$ is in $V$.

Proof. Suppose for a contradiction that a condition $p$ in $\mathbb{P}_{\alpha}^{\prime}$ forces $\dot{X} \subseteq V$ is a set such that for all $A$ in $\left([V]^{<\kappa}\right)^{V}, \dot{X} \cap A \in V$, but $\dot{X}$ is not in $V$.

Let $\theta$ be a regular cardinal much larger than $\mathbb{P}_{\alpha}$ and $\dot{X}$, and let $\mathcal{A}$ denote the structure $\left\langle H(\theta), \in,<_{\theta}, \mathbb{P}_{\alpha}, \sigma\right\rangle$, where $<_{\theta}$ is a well-ordering of $H(\theta)$. Fix an elementary substructure $N$ of $\mathcal{A}$ such that $|N|<\kappa, N \cap \kappa \in \kappa, p$ and $\dot{X}$ are in $N$, and $N$ is in $I A(\mu)$. Then $N^{<\mu} \subseteq N$. By Proposition 3.7 (1), fix $q \leq^{*} p$ such that for any dense subset $D$ of $\left\langle\mathbb{P}_{\alpha}, \leq^{*}\right\rangle$ in $N$, there is $u$ in $N \cap D$ such that $q \leq^{*} u$.

Since $N$ is in $\left([V]^{<\kappa}\right)^{V}$, fix a condition $r \leq q$ in $\mathbb{P}_{\alpha}^{\prime}$ which decides $\dot{X} \cap N$. Let $g: \alpha \rightarrow \operatorname{ADD}(\mu)$ be the partial function such that $\operatorname{dom}(g)=\operatorname{dom}(r) \cap$ Even $\cap N$ and for all $i$ in $\operatorname{dom}(g), r(i)$ is the canonical $\mathbb{P}_{i}$-name for $g(i)$. Since $N^{<\mu} \subseteq N, g$ is in $N$.

Let $D$ be the set of $u$ in $\mathbb{P}_{\alpha}$ such that either $u$ is $\leq^{*}$-incompatible with $p$, or $u \leq^{*} p$ and there are $y_{0}, y_{1} \leq u$ in $\mathbb{P}_{\alpha}^{\prime}$ such that:

(1) for $k=0,1, \operatorname{dom}(g) \subseteq \operatorname{dom}\left(y_{k}\right)$ and for all $i$ in $\operatorname{dom}(g), y_{k}(i)$ is the canonical $\mathbb{P}_{i}$-name for a condition in $\operatorname{ADD}(\mu)$ which extends $g(i)$,

(2) $y_{0} \uparrow$ Odd $=y_{1}\lceil$ Odd $=u \uparrow$ Odd,

(3) there is $a$ such that $y_{0} \Vdash \check{a} \in \dot{X}$ and $y_{1} \Vdash \check{a} \notin \dot{X}$.

By elementarity $D$ is in $N$.

We claim that $D$ is dense in $\left\langle\mathbb{P}_{\alpha}, \leq^{*}\right\rangle$. Let $s$ in $\mathbb{P}_{\alpha}$ be given. If $s$ is $\leq^{*}$ incompatible with $p$ then $s$ is in $D$. Otherwise fix $t \leq^{*} p, s$. Define $w$ by letting 
$w \uparrow \operatorname{Odd}=t \uparrow \operatorname{Odd}, \operatorname{dom}(w) \cap \operatorname{Even}=\operatorname{dom}(g)$, and for all $i$ in $\operatorname{dom}(g), w(i)$ is the canonical $\mathbb{P}_{i}$-name for $g(i)$. Exactly as in the proof of Proposition $3.7(2), w$ is a condition below $t$. Since $w \leq p, w$ forces $\dot{X}$ is not in $V$. In particular, there is $a$ such that $w$ does not decide whether or not $a$ is in $\dot{X}$. So there are $w_{0}, w_{1} \leq w$ in $\mathbb{P}_{\alpha}^{\prime}$ such that $w_{0} \Vdash \check{a} \in \dot{X}$ and $w_{1} \Vdash \check{a} \notin \dot{X}$. Now apply Lemma 3.10 to obtain $y_{0} \leq w_{0}$ and $y_{1} \leq w_{1}$ in $\mathbb{P}_{\alpha}^{\prime}$ such that $y_{0} \uparrow$ Odd $=y_{1}\lceil$ Odd and for all $i$ in $\operatorname{dom}(t) \cap$ Odd, $\mathbb{P}_{i}$ forces $y_{0}(i)=y_{1}(i)$ is below $t(i)$. Define $u$ by letting $u \uparrow$ Even $=t \uparrow$ Even and $u \uparrow$ Odd $=y_{0} \uparrow$ Odd $=y_{1} \uparrow$ Odd. Clearly then $y_{0}, y_{1} \leq u \leq{ }^{*} t \leq{ }^{*} s$ and (1), (2), and (3) are satisfied.

By the choice of $q$, there is $u$ in $D \cap N$ such that $q \leq^{*} u$. Since $q \leq^{*} u, p, u$ and $p$ are $\leq^{*}$-compatible. By elementarity and the definition of $D$ we can fix $y_{0}$ and $y_{1}$ below $u$ in $N$ satisfying (1), (2), and (3). Fix $a$ in $N$ as described in (3). Both $y_{0}$ and $y_{1}$ are compatible with $r$, as follows from (1), (2), and Lemma 3.6, exactly as in the end of the proof of Proposition 3.7 (2). But $r$ decides $\dot{X} \cap N$, so it decides whether or not $a$ is in $\dot{X}$. So $r$ cannot be compatible with both $y_{0}$ and $y_{1}$.

\section{Mitchell's Model}

For the convenience of the reader, we give a sketch of Mitchell's construction of a model with no Aronszajn trees on a successor cardinal ${ }^{1}$, using the framework described in Section 3. None of the results of this section are due to the author. We give several original applications of the iteration schema of Section 3 in [6].

Let $\mu$ be an infinite cardinal such that $\mu^{<\mu}=\mu$, and suppose $\kappa>\mu$ is a regular cardinal such that for all $\zeta<\kappa, \zeta^{<\mu}<\kappa$. Suppose that $\lambda>\kappa$ is a strongly inaccessible cardinal.

We define by induction a forcing iteration $\left\langle\mathbb{P}_{i}, \dot{\mathbb{Q}}_{j}: i \leq \lambda, j<\lambda\right\rangle$ which satisfies (A) - (F) of Section 3. The only thing we need to specify is the value of $\dot{\mathbb{Q}}_{i}$ when $i$ is odd and check that (B) and (C) hold. Suppose $\mathbb{P}_{i}$ is defined for some $i<\lambda$. If $i$ is even, then by $(\mathrm{A})$ we let $\dot{\mathbb{Q}}_{i}$ be a $\mathbb{P}_{i}$-name for $\operatorname{ADD}(\mu)$. If $i$ is odd, let $\dot{\mathbb{Q}}_{i}$ be a $\mathbb{P}_{i}$-name for $\operatorname{CoLL}\left(\kappa, \kappa^{+}\right)$. Then (B) is clear, and (C) is satisfied by Proposition 2.1. By Section 3, the iteration satisfies properties (I) - (IX). By (VII), $\mathbb{P}_{\lambda}$ preserves all cardinals and cofinalities less than or equal to $\kappa$.

Since $\lambda$ is strongly inaccessible, for all $i<\lambda, \mathbb{P}_{i}$ has size less than $\lambda$. It follows by a standard argument that $\mathbb{P}_{\lambda}$ is $\lambda$-c.c. Since we iterate collapses, every cardinal in the interval $(\kappa, \lambda)$ is collapsed to have size $\kappa$. So $\lambda$ is the successor of $\kappa$ after forcing with $\mathbb{P}_{\lambda}$. Also $\mathbb{P}_{\lambda}$ forces $2^{\mu}=\lambda$.

We show that if $\lambda$ is a weakly compact cardinal, then $\mathbb{P}_{\lambda}$ forces that there are no Aronszajn trees on $\lambda=\kappa^{+}$. Suppose for a contradiction there is $p$ in $\mathbb{P}_{\lambda}$ which forces that $\dot{T}$ is an Aronszajn tree on $\lambda$. We may assume that the nodes of $\dot{T}$ are exactly the ordinals below $\lambda$. Therefore without loss of generality we can assume $\dot{T} \subseteq V_{\lambda}$. Let $C$ be the club of $\alpha$ less than $\lambda$ such that $p$ forces $\left(\dot{T} \cap V_{\alpha}\right)^{\dot{G}_{\alpha}}=\dot{T}\lceil\alpha$.

Consider the structure $\left\langle V_{\lambda}, \in, \mathbb{P}_{\lambda}, \Vdash, p, \dot{T}\right\rangle$. This structure models the $\Pi_{1}^{1}$ statement: for all $A$, if $A$ is a $\mathbb{P}_{\lambda}$-name, then for all $q \leq p$ in $\mathbb{P}_{\lambda}, q$ forces $A$ is not a cofinal branch in $\dot{T}$. Since $\lambda$ is weakly compact, there is an inaccessible cardinal $\alpha<\lambda$ in $C$ such that $\left\langle V_{\alpha}, \in, \mathbb{P}_{\alpha}, \Vdash, p, \dot{T} \cap V_{\alpha}\right\rangle$ models the same statement.

Write $\mathbb{P}_{\lambda}=\mathbb{P}_{\alpha} * \dot{\mathbb{P}}_{\alpha, \lambda}$. Then $\mathbb{P}_{\alpha}$ forces that $\dot{\mathbb{P}}_{\alpha, \lambda}$ is equivalent to a forcing iteration satisfying properties (A) - (F) of Section 3. Let $G_{\alpha} * H$ be a generic filter

\footnotetext{
${ }^{1}$ As noted in [7], the consistency of no Aronszajn trees on $\omega_{2}$ is due in part to J. Silver.
} 
for $\mathbb{P}_{\alpha} * \dot{\mathbb{P}}_{\alpha, \lambda}$ which contains $p$. Since $\alpha$ is inaccessible, $\alpha$ is the successor of $\kappa$ in $V\left[G_{\alpha}\right]$. By assumption on $\alpha$, in $V\left[G_{\alpha}\right]$ the tree $S=\left(\dot{T} \cap V_{\alpha}\right)^{G_{\alpha}}$ has no cofinal branch. In $V\left[G_{\alpha} * H\right], T=\dot{T}^{G_{\alpha} * H}$ is a tree on $\lambda$ and $T\lceil\alpha=S$.

Let $c$ be a node on level $\alpha$ of $T$ in $V\left[G_{\alpha} * H\right]$, and let $b=\left\{x \in T: x<_{T} c\right\}$. Then $b$ is a cofinal branch through $S$, and therefore $b$ is not in $V\left[G_{\alpha}\right]$. Suppose $A$ is in $\left(V\left[G_{\alpha}\right]^{<\kappa}\right)^{V\left[G_{\alpha}\right]}$. Then $A \cap b$ is bounded in $S$, so there is $z$ in $b$ such that $x<_{T} z$ for all $x$ in $A \cap b$. But then $A \cap b$ is exactly the set $A \cap\left\{x \in S: x<_{T} z\right\}$, which is in $V\left[G_{\alpha}\right]$. By Property (IX) of Section 3 applied to $\dot{\mathbb{P}}_{\alpha, \lambda}, b$ is in $V\left[G_{\alpha}\right]$, which is a contradiction.

\section{REFERENCES}

[1] U. Abraham and S. Shelah. Forcing closed unbounded sets. J. Symbolic Logic, 48(3):643-657, 1983.

[2] J. Baumgartner. Iterated forcing. In Surveys in Set Theory. Cambridge Univ. Press, 1983.

[3] M. Foreman and M. Magidor. Large cardinals and definable counterexamples to the continuum hypothesis. Ann. Pure Appl. Logic, 76(1):47-97, 1995.

[4] M. Gitik and J. Krueger. Approachability at the second successor of a singular cardinal. Submitted.

[5] J. Krueger. Internally club and approachable for larger structures. Submitted.

[6] J. Krueger. Some applications of mixed support iterations. Submitted.

[7] W. Mitchell. Aronszajn trees and the independence of the transfer property. Ann. Math. Logic, $5: 21-46,1972 / 73$.

[8] W. Mitchell. On the Hamkins approximation property. Ann. Pure Appl. Logic, 144(1-3):126129,2006

Department of Mathematics, University of California, Berkeley

E-mail address: jkrueger@math.berkeley.edu

URL: http://www.math.berkeley.edu/ jkrueger 